\title{
Numerical Investigation on Various Heat Exchanger Performances to Determine an Optimum Configuration for Charge Air Cooler, Oil and Water Radiators in F1 Sidepods
}

\author{
Pierre Salmon ${ }^{\mathrm{a}}$, László Könözsy ${ }^{\mathrm{b}, *}$, Clive Temple ${ }^{\mathrm{a}}$, Stuart Grove ${ }^{\mathrm{a}}$ \\ ${ }^{a}$ Centre for Advanced Vehicle Engineering, Cranfield University, Cranfield, Bedfordshire, MK43 OAL, United Kingdom \\ ${ }^{b}$ Centre for Computational Engineering Sciences, Cranfield University, Cranfield, Bedfordshire, MK43 OAL, United Kingdom
}

\begin{abstract}
The present work focuses on a three-dimensional CFD approach to predict the performance of various heat exchangers in conjunction with non-isothermal transitional flows for motorsport applications. The objective of this study is to determine the heat transfer, pressure drop and inhomogeneous flow behaviour for distinct heat exchangers to identify an optimum configuration for the charge air cooler, water and oil radiators placed in the sidepods of a formula one (F1) car. Therefore, a comprehensive analysis of various heat exchanger configurations has been carried out in this work. In order to assess the reliability of the obtained results, a mesh sensitivity study along with additional parametric investigations have been performed to provide numerical parameters predicting accurately a) the heat transfer rate at the fluid-solid interface and b) the sporadic separation. As a result of the performed validation procedure in this study, the aerodynamic- and thermal boundary layer development along with the convective characteristics of the air flow have been captured accurately near to the heated surface. The characterization of a heat exchanger core and a core configuration in a closed domain is also possible with this procedure. The presented three-dimensional CFD approach could overcome the difficulties of macroscopic heat exchanger and porous media methods for F1 applications, because it can be used to predict the heat transfer and pressure drop related to the mass flow rate correlation curves. The contribution of fins to the total heat transfer rate has been predicted theoretically, and application benchmark test cases have been presented to analyze five different heat exchanger configurations in accordance with the 2014 formula one technical regulations. The numerical data extracted directly from three-dimensional CFD simulations can be used in the sidepod design process of the external cooling system of F1 engines.
\end{abstract}

Keywords: heat exchangers, non-isothermal transitional flows, formula one, charge air cooler, sidepod, fin

\section{Introduction}

Heat exchangers in automotive and motorsport applications often operate at flow velocities corresponding to Reynolds numbers indicative of the laminar-to-turbulent transitional flow regime [1]. Therefore, robust and accurate numerical simulations are required to predict gradients of all flow field variables correctly. High-resolution meshes and advanced engineering turbulence modelling approaches are capable of capturing sporadic separations and adverse pressure gradients in the boundary layer of heated surfaces. In the field of computational fluid dynamics (CFD), traditional numerical procedures usually employ the porous media method where the implementation of the heat transfer to mass flow rate curves are pre-determined [2, 3]. Due to the constraint of semi-empirical correlations, the porous media approach could loose its accuracy related to those real world applications where sporadic separation and/or nonadiabatic pressure drop is observed. The non-linear nature of heat transfer and pressure drop requires a more accurate simulation approach than the use of semi-empirical correlations or the porous media method. Therefore, the present work focuses on an advanced computational engineering procedure to determine

\footnotetext{
${ }^{*}$ Corresponding author

Email address: laszlo.konozsy@cranfield.ac.uk (László Könözsy)
}

performance curves of various heat exchanger configurations relying on direct three-dimensional CFD heat transfer simulations for a formula one (F1) car sidepod.

An intensive development on different heat exchanger design including air-cooled ones was carried out over the past fifty years. Application of direct CFD methods is playing an important role, because the performance of modern computers is highly increased. Bhutta et al. [4] carried out a detailed review on advanced CFD applications in various heat exchangers design. Wang et al. [5] focused on the investigation of the data reduction method for air-side performance of fin-and-tube heat exchangers, because this method for the air-side heat coefficients were not relying on a consistent approach in the literature. Xie et al. [6] performed numerical parametric studies and obtained multiple correlations on air-side heat transfer and friction characteristics when large number of large-diameter tube rows were considered. Borrajo-Peláez [7] carried out a threedimensional CFD study to compare the air-side model with the air/water model for a plain fin-and-tube heat exchanger. Koplow [8] proposed a fundamentally novel approach to aircooled heat exchangers highlighting that CFD data would be very useful in the transitional flow regime for device optimization purposes. Fugmann et al. [9] evaluated the performance of air-based heat rejection system related to simulation methods. Erek et al. [10] presented CFD simulation data on the influ- 
ences of the fin geometry on heat transfer and pressure drop for a plate fin-and-tube heat exchanger. Bhuiyan et al. [11] performed a three-dimensional CFD analysis on plate fin-and-tube heat exchangers providing reliable numerical data in the transitional flow regime. Bhuiyan et al. [12] investigated the effects of performance parameters for turbulent flows as well.

Heat exchangers use fins to enhance heat transfer by increasing the heat transferring surface area and inducing turbulent mixing [13, 14]. Louvered fin geometry aids the reestablishment of high temperature gradients near the surfaces which enhance solid to fluid thermal energy exchange [1522]. The work of Salim and Cheah [23] suggests that a numerical procedure for simulating heat transfer should model the near-wall region accurately to capture the large gradients of the solution variables. Therefore, an advanced grid generation strategy for complex heat exchanger geometries has to incorporate prismatic layered elements aligned to all surfaces with smooth transition into the upper tetrahedral mesh region.

Huang et al. [24] stated that the porous media method admittedly gives poor correlation to test results under certain conditions when the energy equation is not resolved properly.Therefore, a three-dimensional CFD approach is recommended to perform a conclusive analysis on heat exchanger performances including the visualisation of vortical structures, pressure gradients, temperature distributions and separation events which are present in the physical and computational domain [25]. An adequate prediction of relevant process parameters gives an opportunity for the optimization of heat exchanger configurations, which can yield power-unit performance, packaging and drag reduction benefits for F1 car applications.

The objective of the present 3D CFD study is to identify an optimum configuration for the charge air cooler, water and oil radiators placed in F1 sidepods in accordance with the $2014 \mathrm{~F} 1$ technical regulations (see Figure 1). The sidepod is an external bodywork of F1 cars related to the external cooling system of $\mathrm{F} 1$ engines which cannot be found in common vehicles [26].

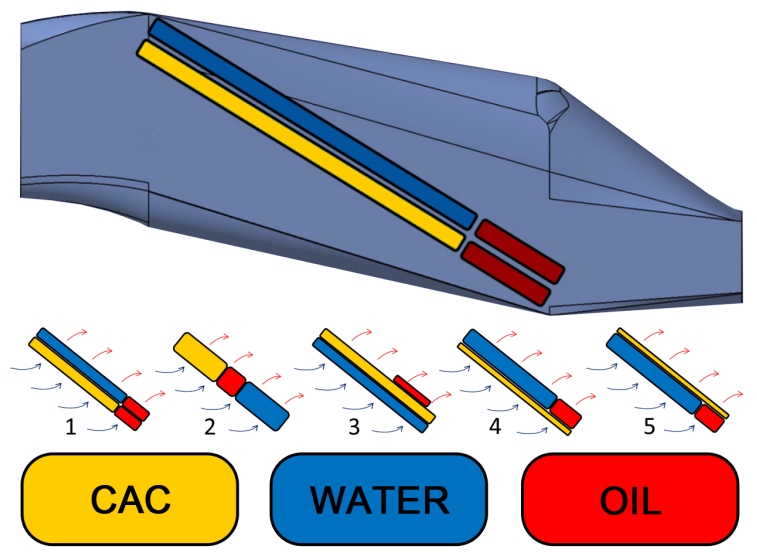

Figure 1: Five distinct heat exchanger configurations to determine an optimum one for the charge air cooler, water and oil radiators placed in the sidepods.

In this paper, the aforementioned configuration is defined and referred as the relative placement of the heat exchangers with respect to each other in the sidepod domain (see Figure11).
The outer air-side of the heat exchanger configurations has been considered which is also called as the cold side, because it contains the cooling air. Sporadic separation in the transitional flow regime and vortical flow structures exiting the cooling cores have an impact on the pressure gradients at the sidepod outlets which directly affect the performance of vehicle aerodynamic surfaces. The presented three-dimensional CFD approach overcomes this problem and predicts the vortical flow structures and heat transfer rates from a heat exchanger configurations that accounts for real world applications in the design of F1 sidepods.

In Configurations 1 and 2, the shape of the sidepod inlet duct is triangular and the heat exchanger shape is rectangular as well as the low angle of incidence between the inlet air flow and the cores. In Configuration 3 , the oil radiator is placed at the bottom of the sidepod domain in a third tier to provide an additional aerodynamic resistance at the bottom of the sidepod. This radiator placement helps to redistribute the air mass flow uniformly through the cores to overcome the technical difficulties encountered in Configurations 1 and 2. The redistribution of mass flow rate across the cores allows for higher net heat transfer rates and a reduction of the air flow turbulent intensity in the rear section of the sidepod domain. This effect is advantageous, because a uniform velocity distribution through the cores can lead to a reduction in the pressure drop. In Configurations 4 and 5 , the oil and water radiators are placed in a similar position to the 2013 generation of formula one cars with charge air cooler placed upstream and downstream of the radiators.

To evaluate the performance of different heat exchanger configurations for motorsport applications, the accuracy and reliability of engineering turbulence models has to be assessed in the laminar-to-turbulent transitional flow regime. The uncertainty of numerical approaches to transitional flows can be predicted through a mesh sensitivity study related to each employed engineering turbulence model. To obtain physically correct numerical results for non-isothermal transitional flows through complex heat exchanger geometries, it is necessary to verify the implementation of the initial and boundary conditions and develop model parameters on an appropriate benchmark problem. For non-isothermal transitional flows, the heat transfer rate can be calculated theoretically from the hot internal fluid to the cold outer fluid in a thin tube of a heat exchanger, which is considered as a benchmark for model validation purposes [27- [32].

To investigate the performance characteristics of a cooling system of a formula one car, the contribution of the fins to heat transfer has been predicted theoretically and the resultant Nusselt numbers have been calculated for each flow condition based on the CFD simulation results. Due to the competitive nature of the motorsport industry, there is a lack of experimental and numerical data available in the literature for the performance and design of complex heat exchanger configurations in current F1 applications. It is increasingly important to find the correct and optimum heat exchanger configuration, because heat rejection requirements have gone up for the new engines, but the packing and aerodynamic constraints are remained the same. This work provides three-dimensional CFD simulation data for five different heat exchanger configurations which can be used in the design process of the external cooling system of F1 engines. 


\section{Governing Equations and Solution Methodology}

For modelling incompressible, non-isothermal transitional flows, the mass, momentum and energy conservation equations are solved along with different Reynolds-Averaged NavierStokes (RANS) engineering turbulence models. The conservation of mass can be written with index notation as

$$
\frac{\partial u_{i}}{\partial x_{i}}=0
$$

where $u_{i}$ is the velocity vector. To compute the viscous shear drag induced pressure drop across the domain, the Reynolds' conservation of momentum equation can be considered as

$$
\rho u_{j} \frac{\partial u_{i}}{\partial x_{j}}=\rho g_{i}-\frac{\partial p}{\partial x_{i}}+\mu \frac{\partial^{2} u_{i}}{\partial x_{j} \partial x_{j}}+\frac{\partial}{\partial x_{j}}\left(-\rho \overline{u_{i}^{\prime} u_{j}^{\prime}}\right),
$$

where $\rho$ is the fluid density, $g_{i}$ is the vector of the conservative gravity field, $p$ is the hydrodynamic pressure, $\mu$ is the dynamic viscosity of the fluid, and $u_{i}^{\prime}$ is the velocity fluctuation vector. The elements of the Reynolds' stress tensor $R_{i j}=-\rho \overline{u_{i}^{\prime} u_{j}^{\prime}}$ in Eq. (2) can be modelled by applying engineering turbulence models for the closure problem. The energy equation is solved to take into account the thermal energy exchange from the heated surfaces to the cooling air, including the viscous dissipation as

$$
\begin{gathered}
\rho c_{p} u_{j} \frac{\partial T}{\partial x_{j}}=u_{j} \frac{\partial p}{\partial x_{j}}+\frac{\partial}{\partial x_{j}}\left(\lambda \frac{\partial T}{\partial x_{j}}\right)- \\
-\rho c_{p} \frac{\partial}{\partial x_{j}}\left(\overline{u_{j}^{\prime} T^{\prime}}\right)+\varphi_{D}+\rho \varepsilon+\varphi_{S}
\end{gathered}
$$

where $c_{p}$ is the specific heat capacity, $\lambda$ is the thermal conductivity, $T^{\prime}$ is the local temperature fluctuation, $\varphi_{D}$ is the viscous dissipation function, $\varepsilon$ is the turbulent energy dissipation, and $\varphi_{S}$ represents heat sources in the computational domain. Each turbulence model predicts the Reynolds' stress distribution with different mathematical assumptions through modelling velocity and temperature fluctuations. Therefore, the accuracy and performance of seven turbulence models have been assessed and analyzed to select the most accurate and efficient modelling approach to various complex heat exchanger configurations.

The heat transfer rate can be increased through louvered fin geometry and vortex generators to enhance turbulent mixing and high temperature gradients near the surfaces [13, 14, 33]. To compute the heat transfer from the fins, temperature gradients along the length of the fins have to be expressed with variable convection coefficients or a fin efficiency factor [34]. The contribution of fins influences the overall performance of a heat exchanger which can be determined by using engineering formulae for the fin effectiveness parameter $m$, the surface area of the base tube $A_{b a s e}$ and the thermal efficiency of a fin $\eta_{\text {fin }}$. The fin effectiveness parameter $m$ can be calculated [34] by

$$
m=\sqrt{\frac{2 \cdot h_{\text {base }}}{k \cdot \delta_{\text {fin }}}},
$$

where $k$ is the thermal conductivity of aluminium taken as 237 $W /(m K)[34], \delta_{\text {fin }}$ is the fin thickness, and the local convective heat transfer coefficient of the base tube can be predicted as

$$
h_{\text {base }}=\frac{\dot{Q}_{\text {base }}}{A_{\text {base }}\left(T_{\text {base }}-T_{\text {air }}\right)},
$$

where $\dot{Q}_{\text {base }}$ is the heat transfer rate of the thin tube obtained from the CFD simulation, $T_{\text {base }}$ is the temperature of the base tube and $T_{\text {air }}$ is the air temperature at $298 \mathrm{~K}$. It is important to note that $\dot{Q}_{\text {base }}$ is depending on the Reynolds number, temperature, surface area and material properties. The base tube Nusselt numbers $\mathrm{Nu}$ can also be computed relying on the CFD simulation results by using Eqs. (4) and (5) as

$$
N u=\frac{h_{\text {base }} \cdot D_{h}}{k},
$$

where $D_{h}$ is the hydraulic diameter. This direct method of calculating the contribution of fins to the total heat transfer could overcome the difficulties of the porous media method, because there is no need for any prior knowledge on the fins in this case. Furthermore, this direct approach incorporates the theoretically predicted local convective heat transfer coefficients (5) with the computation of Nusselt numbers (6) based on the CFD simulations. By using all of these values for each flow velocity condition, the thermal efficiency of a fin is predicted [34] as

$$
\eta_{f i n}=\frac{\dot{Q}_{\text {fin }} \text { real }}{\dot{Q}_{\text {finideal }}}=\frac{\tanh \left(m \cdot l_{\text {fin }}\right)}{m \cdot l_{\text {fin }}} .
$$

where $\dot{Q}_{\text {fin }_{\text {real }}}$ is the sum of the real heat transfer rates of the fins, $\dot{Q}_{\text {fin }_{\text {ideal }}}$ is the sum of the maximum heat transfer rates of the fins, and $l_{\text {fin }}$ is the length of the fin. The total performance of a heat exchanger in one data point can be calculated by

$$
\dot{Q}_{t o t}=h_{\text {base }} \cdot\left(A_{\text {base }}+A_{\text {fins }} \cdot \eta_{\text {fin }}\right) \cdot\left(T_{\text {base }}-T_{\text {air }}\right),
$$

where each data point represents one heat exchanger tube with a set surface temperature at a specified inlet flow velocity. Relying on this approach in conjunction with CFD simulations, the heat transfer rate and pressure drop curves can be predicted with the advantage of capturing the three-dimensional flow structures inside the sidepod. The heat transfer rate and pressure drop curves have been extracted from the data set of the three-dimensional CFD simulations. For post-processing purposes, User-Defined Functions (UDFs) by using the ANSYSFLUENT environment and MATLAB scripts have been implemented to investigate different heat exchanger configurations. A second-order upwind discretization scheme has been used for computing the convective flux terms [4]. For the pressurevelocity coupling, the PRESTO (PREssure STaggering Option) algorithm has been selected as it provides an improved numerical accuracy and robustness for solving non-isothermal transitional flows in a strongly curved domain. The V6 internal combusion engine simulation is performed by using the AVL BOOST engine simulation software tool, and we refer to the V6 engine design and simulation carried out in [35]. 


\section{Mesh Sensitivity Study with the Assessment of Seven Turbulence Models for Transitional Flows in a Thin Tube}

The primary target of mesh sensitivity and parametric studies is to determine the total heat transfer rate $(8)$ from the hot internal fluid to the cold outer fluid. The numerically predicted heat transfer rates can be compared to the theoretically calculated value of $26.22 \mathrm{~W}$ [34, 36], to assess the accuracy of seven engineering turbulence models in a thin tube of a heat exchanger. The mesh refinement study has been carried out on a fluid domain of a $0.5 \mathrm{~m}$ long tube with inner and outer diameters of $19 \mathrm{~mm}$ and $20 \mathrm{~mm}$, respectively. The air flow velocity of $4 \mathrm{~m} / \mathrm{s}$ was considered with an inlet temperature of $118.5^{\circ} \mathrm{C}$ on the internal domain, with air flowing transversely over the tube with an inlet temperature of $20^{\circ} \mathrm{C}$ and inlet velocity of 4 $\mathrm{m} / \mathrm{s}$ in the outer domain. The internal air flow is transitional at the Reynolds number of 3295 which means that the laminar-toturbulent velocity distribution is not fully-developed yet [37]. It is crucial to determine the air flow behaviour in the transitional flow regime accurately, because it has an influence on the net thermal energy exchange from the hot inner fluid through the wall to the cold outer fluid. In addition to this, separation events influence the flow structure and temperature gradients near the heated wall surfaces of heat exchangers, which in turn influence the local heat transfer rate. Therefore, it is important to model the flow convection and diffusion characteristics accurately to gain confidence in the reliability of CFD simulation results.

The mesh refinement consisted of six different grid-levels (see Table 11) and parametric studies have been performed by employing seven different engineering turbulence models (see Table 2 to select the most accurate modelling approach for simulating various heat exchanger configurations in Section 4 To determine the optimum number of grid cells along with the most suitable turbulence model to compute the heat transfer rate accurately, the reliability study can be divided into two parts. In the first stage, the mesh density was systematically increased by reducing the volume element and surface sizing dimensions, and the accuracy of the Transitional SST (Shear Stress Transport) turbulence model is assessed by predicting the heat transfer rate. Each mesh refinement step led to increased accuracy of the simulation results with an improved prediction of the heat transfer rate value. It can be seen that this turbulence model requires approximately 22 million cells to predict the heat transfer rate close to the theoretical value of $26.22 \mathrm{~W}$ (see Table 1). To reduce the computational cost and assess the reliability of this transitional model, it is necessary to evaluate the performance of other turbulence models on a coarser mesh (see Table 2).

Table 1: Results of mesh refinement on six different grid-levels.

\begin{tabular}{lllll}
\hline Grid & Meshing Techniques & Number of Cells & Heat Transfer Rate and Rel. Error \\
\hline 1 & Default Settings with Fine Sizing & 305161 & $30.74 \mathrm{~W}$ & $17.23 \%$ \\
2 & Sizing Relevance set to 100\% & 1145778 & $30.78 \mathrm{~W}$ & $17.39 \%$ \\
3 & Surface Sizing to 0.1mm & 2417092 & $30.29 \mathrm{~W}$ & $15.52 \%$ \\
4 & Surface Inflation, 10 Layers & 3262075 & $27.47 \mathrm{~W}$ & $4.76 \%$ \\
5 & Inner Fluid Body Sizing to 0.1 $\mathrm{mm}$ & 11084992 & $28.06 \mathrm{~W}$ & $7.01 \%$ \\
6 & Surface Sizing to 0.05mm & 22206149 & $26.51 \mathrm{~W}$ & $1.1 \%$ \\
\hline
\end{tabular}

In the second stage of the reliability study, grid-level 4 has been chosen as a coarser baseline mesh to evaluate the accuracy of other turbulence models in predicting the heat transfer rate compared to the theoretical value (see Table 22. The reason for this choice is that the optimum number of computational cells for this thin tube problem can be considered between 3 and 4 million elements, because the results does not change significantly between grid-level 4 and 6 . Note that the inclusion of a surface inflation layer in the meshing procedure between grid-level 3 and 4 improved the capability of capturing thermal and aerodynamic boundary layers as well as adverse pressure gradients in the near-wall region (see Table 1 ).

\begin{tabular}{llc}
\multicolumn{3}{c}{ Table 2: Results of parametric study by using seven turbulence models. } \\
\hline \multicolumn{2}{l}{ Engineering Turbulence Model } & Heat Transfer Rate and Rel. Error \\
\hline 1. Standard $k-\varepsilon$ & $61.01 \mathrm{~W}$ & $132.68 \%$ \\
2. Re-Normalization Group (RNG) $k-\varepsilon$ & $59.84 \mathrm{~W}$ & $128.22 \%$ \\
3. Realizable $k-\varepsilon$ & $45.99 \mathrm{~W}$ & $75.40 \%$ \\
4. Transitional $k k l-\omega$ & $33.78 \mathrm{~W}$ & $28.83 \%$ \\
5. Standard $k-\omega$ & $33.86 \mathrm{~W}$ & $29.13 \%$ \\
6. $k$ - $\omega$ SST & $32.84 \mathrm{~W}$ & $25.24 \%$ \\
7. Transitional SST & $27.47 \mathrm{~W}$ & $4.76 \%$ \\
Theoretically Predicted Reference Value & $26.22 \mathrm{~W}$ &
\end{tabular}

It can be seen in Tables 2 and 3 that the Transitional SST model most accurately predicts the heat transfer rate compared to the theoretically calculated value of $26.22 \mathrm{~W}$. By comparing the Realizable k- $\varepsilon$ to the Transitional SST model for six successively refined meshes, the Transitional SST model achieves lower residuals after 3000 iterations on a coarser mesh for the continuity and energy equations. These results indicate that the Transitional SST model would be more accurate and robust for solving the governing equations of non-isothermal transitional flow problems in complex geometries (see Tables 2 and 3 .

Table 3: Convergence residuals of mesh refinement and reliability studies.

\begin{tabular}{lllll}
\hline & Continuity Equation & Continuity Equation & Energy Equation & Energy Equation \\
\hline Grid-Level & Realizable k- $\varepsilon$ & Transitional SST & Realizable k- $\varepsilon$ & Transitional SST \\
\hline 1 & $1.86 \mathrm{e}-3$ & $5.55 \mathrm{e}-5$ & $1.01 \mathrm{e}-6$ & $2.28 \mathrm{e}-7$ \\
2 & $2.54 \mathrm{e}-3$ & $1.60 \mathrm{e}-5$ & $7.21 \mathrm{e}-7$ & $9.14 \mathrm{e}-8$ \\
3 & $1.50 \mathrm{e}-3$ & $1.14 \mathrm{e}-5$ & $4.09 \mathrm{e}-7$ & $7.95 \mathrm{e}-8$ \\
4 & $4.94 \mathrm{e}-6$ & $8.10 \mathrm{e}-6$ & $7.57 \mathrm{e}-8$ & $7.53 \mathrm{e}-8$ \\
5 & $6.60 \mathrm{e}-6$ & $1.20 \mathrm{e}-6$ & $7.59 \mathrm{e}-8$ & $7.36 \mathrm{e}-8$ \\
6 & $6.90 \mathrm{e}-6$ & $5.25 \mathrm{e}-6$ & $1.07 \mathrm{e}-7$ & $6.89 \mathrm{e}-8$ \\
\hline
\end{tabular}

\section{Results and Discussions}

In this Section, the relative heat exchanger placement in an F1 car sidepod domain, which influences the holistic performance of formula one cooling systems, has been investigated for five distinct configurations (see Figure 1). The fin effects on heat transfer were studied extensively by Chang and Wang [13], and Oliet et al. [38] showed that the increment of the fin density increases the heat transfer rate at the cost of the increased pressure drop. In the present work, the air inlet temperature has been set to $298 \mathrm{~K}$ and the wall temperatures of the charge air cooler, oil and water radiators have been set to $377 \mathrm{~K}, 385.5$ $K$ and $380.5 K$, respectively. The inlet flow velocity has been ranged stepwise from $0.1 \mathrm{~m} / \mathrm{s}$ to $90 \mathrm{~m} / \mathrm{s}$. An example computational mesh of the heat exchanger Configuration 3 with approximately 25 million elements is shown in Figure 2. A prismtetrahedral hybrid mesh has been generated along with an inflation layer applied to the domain outer surface including the 
charge air cooler, water and oil radiator walls. The first layer thickness was $0.05 \mathrm{~m}$ with the number of maximum layer 10 and with $10 \%$ growth to capture the boundary layer flow, separation phenomenon and heat transfer rates accurately.

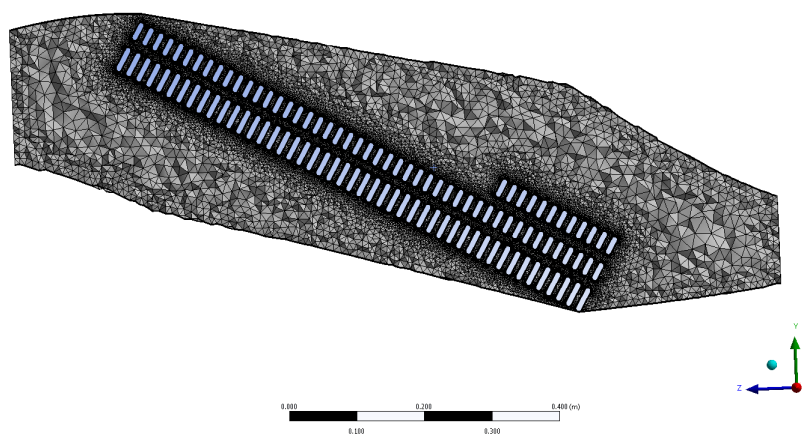

Figure 2: Computational mesh of the heat exchanger Configuration 3 placed in the sidepod with approximately 25 million elements.

To determine an optimum heat exchanger configuration for the charge air cooler, water and oil radiators placed in the sidepod of formula one cars, the highest net heat transfer rate has to be identified which has an impact on the performance of the entire configuration. After extracting all computational data from the three-dimensional CFD simulations, the pressure drop and heat transfer rate versus mass flow rate curves have been nondimensionalized based on their maximum values. Therefore, each curve tends to the maximum value of one in Figures 3 and 5 . The use of dimensionless pressure drop and heat transfer rate are advantageous in these cases, because it is possible to validate the obtained CFD simulation results and all dimensionless curves can be compared to other studies [24, 38,-40] when slightly different mass flow rates and heat exchanger sizes were used by other authors relevant to the present study.

The pressure drop across a heat exchanger increases exponentially with an increase of the air flow velocity (see Figure 3 . The area ratio between the sidepod and the heat exchanger inlet faces governs the pressure gradient within the sidepod. The inlet shape of the sidepod relative to the heat exchanger core shape and the flow velocity incidence angle results in a nonuniform mass flow rate distribution biased toward the lower inboard region. The non-uniform volume expansion of the duct results in a non-uniform mass flow rate through the cores which causes non-uniform local pressure drops and heat transfer rate distributions from the tubes. It means that any non-uniformity of the air flow has an impact on the sub-optimal system performance which can be explained by the non-linear relationship between the pressure drop and the heat transfer rate related to mass flow rate [5, 7, 11, 12, 16,-18, 41,-49]. The heat exchanger applications investigated by Huang et al. [24], Oliet et al. [38] and Yang et al. [39] are similar to the numerically simulated cases in the present work. As an example, for Configuration 2, the pressure drop increases across the mass flow rate range and exhibits non-linear behaviour which corresponds to the aforementioned relevant studies [24, 38, 39] (see Figure 3). Relying on the simulation results for each configuration, it can be seen in Figure 4 that the increase in pressure drop versus mass flow rate curves does not necessarily exhibit a smooth theoretically expected exponential behaviour. The reason is that the variation in pressure drop is a result of the time-dependent and sporadic separations that occurs on the rearward facing surfaces of the heat exchanger cores in the laminar-to-turbulent transitional flow regime.

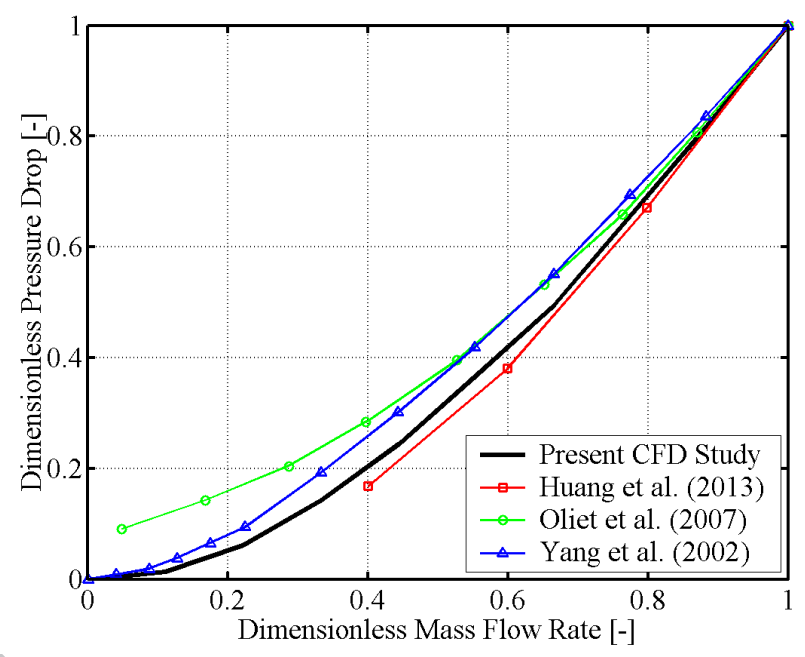

Figure 3: Dimensionless pressure drop [-] versus dimensionless mass flow rate [-] curve of the heat exchanger Configuration 2 compared to other studies.

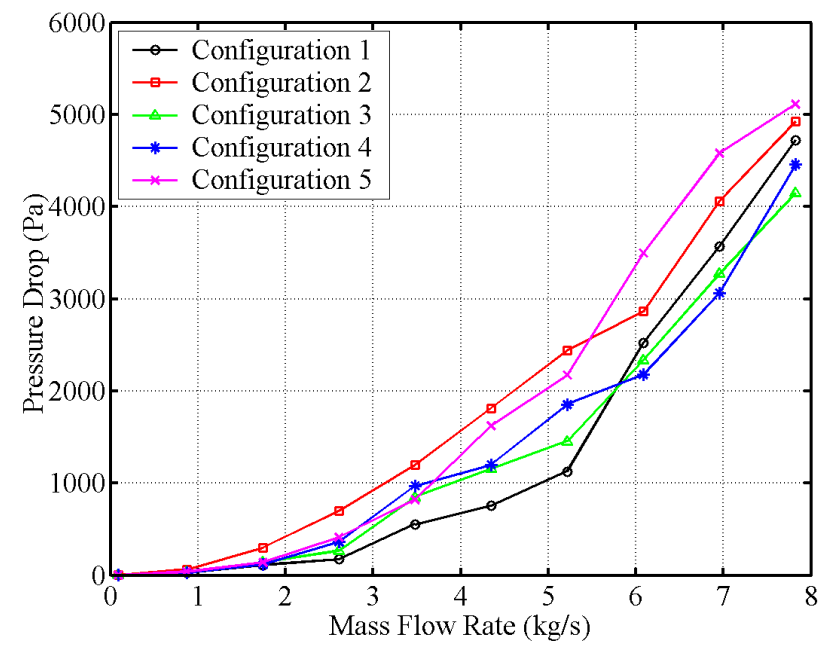

Figure 4: Pressure drop $(\mathrm{Pa})$ versus mass flow rate $(\mathrm{kg} / \mathrm{s})$ curves of distinct heat exchanger configurations.

The simulation results of Configuration 1 showed that the air tended to flow down across the inlet face resulting in a bias of mass flow towards the lower section of the cores. This is due to the fact that the oil radiator of Configuration 1 is placed below the water radiator and charge air cooler in order to evaluate the vertical heat transfer distribution. Configuration 2 is an 
example of a parallel stack arrangement in the sidepod where the longer cores result in a well-developed aerodynamic and thermal boundary layers which lead to reduced heat transfer rates near the rear part of the tubes. This has the effect of reducing the operative flow area, where the cooling air is able to flow with a uniform free-stream velocity, will lead to an aerodynamic resistance at the inlet section. Therefore, a higher pressure drop for a lower mass flow rate range can be seen for Configuration 2 in Figure 4 In Configuration 3, the placement of the oil radiator low in a third tier balances the aerodynamic resistance across the core stack and the uniformity of the resultant mass flow rate, which contributes to the overall heat transfer, pressure drop and sidepod outlet flow conditions. Configuration 5 yields a higher pressure drop than Configuration 4, because the longer cores in the front tier lead to a local flow rate increase and a higher pressure drop across the shorter rear cores. The results suggest that the optimum configuration for a minimum pressure drop penalty perspective could be either Configurations 1,3 or 4 as they yield the lowest pressure drops across the entire mass flow rate range (see Figure 4).

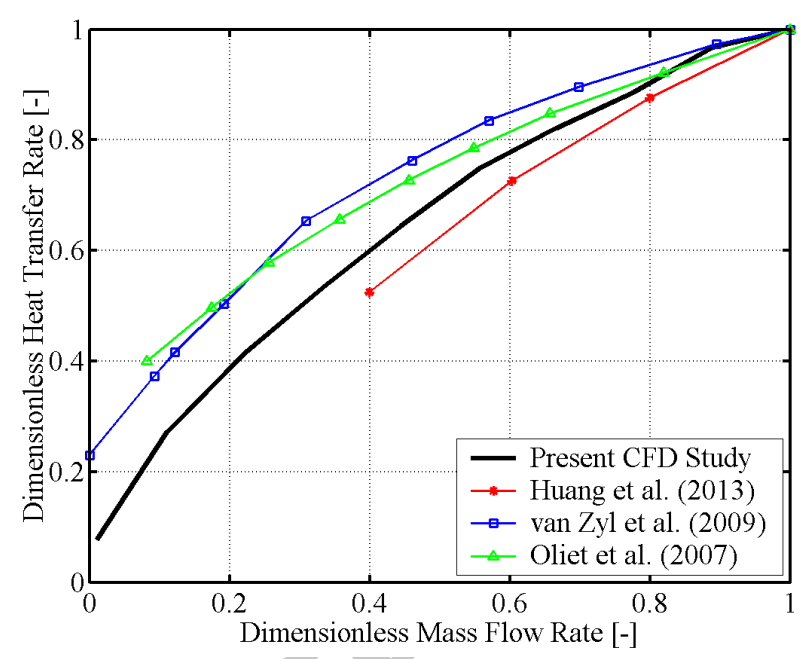

Figure 5: Dimensionless heat transfer rate [-] versus dimensionless mass flow rate $[-]$ curve of the heat exchanger Configuration 4 compared to other studies.

The primary performance metric of a heat exchanger is the ability to reject thermal energy per unit time for a cooling air mass flow rate. A higher heat rejection rate per unit volume of a heat exchanger core is important to motorsport applications, because this allows for smaller heat exchangers and tighter packaging of the rear body-work to the center line of the car, which improves the rear-end aerodynamic efficiency of the vehicle. Therefore, the optimum heat exchanger configuration is that one which rejects the highest rate of thermal energy per unit of cooling air mass flow rate and for the lowest pressure drop. The heat rejection curves published by Huang et al. [24], van Zyl et al. [40] and Oliet et al. [38] are relevant to the present study, because they investigated similar automotive applications. For Configuration 4 , the numerically predicted heat rejection rates have been compared to other studies [24, 38, 40], where coher- ency is found. The variation in these results can be explained by the unique installations and fin geometry (see Figure 5).

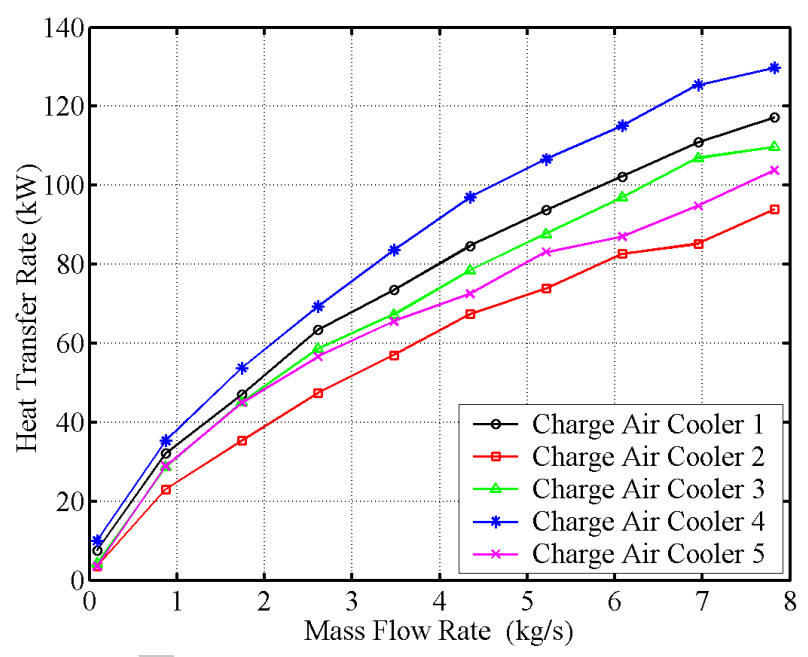

Figure 6: Charge air cooler heat transfer rate $(\mathrm{kW})$ versus mass flow rate $(\mathrm{kg} / \mathrm{s})$ correlation curves.

Configuration 4 yields the highest charge air cooler heat transfer rate throughout the specified inlet mass flow rate range (see Figure 6). This is due to the fact that the charge air cooler is placed upstream and it receives the cooling air at its nominal temperature by having a thinner section but larger projected face area. This result is supported by the obtained simulation data for Configuration 5 where the charge air cooler is placed downstream of the radiators which receives a slightly warmer air. It means that this charge air cooler has a lower net surface to air temperature difference compared to the charge air cooler placed in Configuration 4 even though both charge air coolers have the same shape and geometrical size. It can also be seen in Figure 6 that Configuration 2 has the lowest heat transfer rate, because it has longer charge air cooler cores near the top of the stack, which receives less air flow, and a thermal boundary layer develops near the rear of the core. The heat transfer rate slightly decreases when the mass flow rate increases from 6 to 7 (see Figure 7), because there is an alteration in the mass flow rate distribution through the cores which occurs due to the transient nature of separation along the longer cores. By placing a relatively cooler heat exchanger upstream, more thermal energy can be transferred, because the net surface to air temperature difference across the entire cooling system is optimized for the holistic heat transfer from a given heat exchanger configuration. This design strategy was employed for turbocharged engines in the automotive industry over recent years. When analyzing the effect of the thermal solution on the overall performance of the entire configuration, it can also be seen that Configuration 4 has the highest net heat transfer rate compared to other investigated configurations in this study (see Figures 6and 7).

The underlying physics of the numerical results can be explained by the heat exchanger surface to the cooling air temperature difference across the holistic heat exchanger config- 


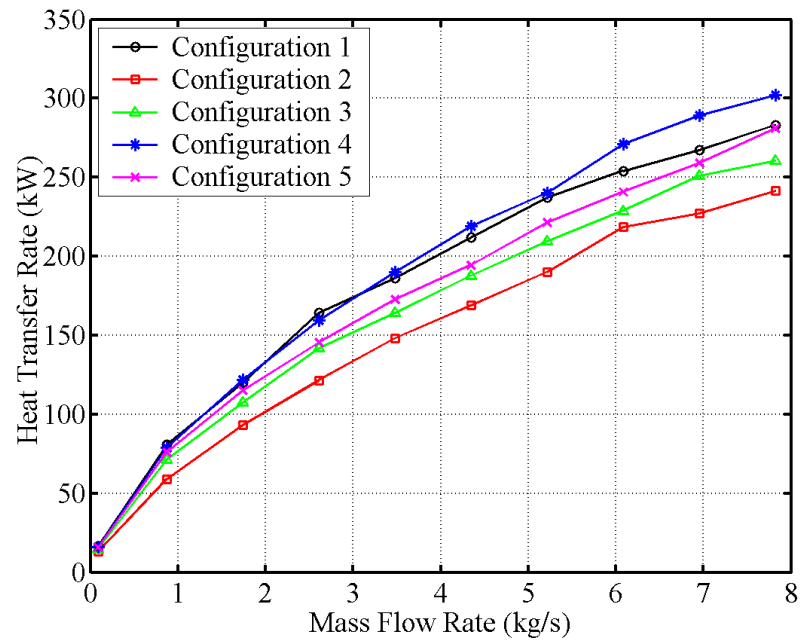

Figure 7: Heat transfer rate $(\mathrm{kW})$ versus mass flow rate $(\mathrm{kg} / \mathrm{s})$ correlation curves of distinct heat exchanger configurations.

uration. In Configuration 4, the charge air cooler is placed in front of the hotter oil- and water radiators which has a relatively lower surface temperature, where the thermal energy is transferred by the charge air cooler in the front section of this configuration. Therefore, the amount of thermal energy transferred to the ambient temperature cooling air is relatively less compared to other configurations. Once the cooling air has passed through the first core, it will have an elevated temperature, which determines the cooling air to the heated surface temperature difference of the next heat exchanger surface downstream. By placing the heat exchanger with the lowest temperature upstream, it allows for the hotter rear section of the heat exchanger matrix to transfer more thermal energy to the warmed up cooling air, thus the resultant net heat transfer rate to the air will be increased. In the other way around, as it can be seen in the case of Configuration 5, the cooling air has more energy to be transferred and it passes through the first tier due to a higher surface to air temperature difference for the liquid radiators. Then, the second tier of this configuration cannot transfer the same amount of thermal energy, due to having a lower surface to air temperature difference. However, in Configuration 4 , the hotter surfaces are placed in the second tier of the heat exchanger stack, thus the holistic surface to air temperature difference is higher, therefore, the overall system heat transfer rate is the highest over the range of the cooling air mass flow rate. Thus, the numerical results of the present study suggest that it is beneficial to place thinner cores with larger inlet face areas in a series stack arrangement with a front to back order which is determined by an increasing surface temperature hierarchy. It can also be concluded that Configuration 4 is the optimum heat exchanger configuration for charge air coolers. This is due to the fact that it rejects the highest rate of thermal energy from the charge air cooler and from the total heat exchanger configuration without a relatively severe pressure drop penalty.

The metric of heat rejection divided by pressure drop is eval-

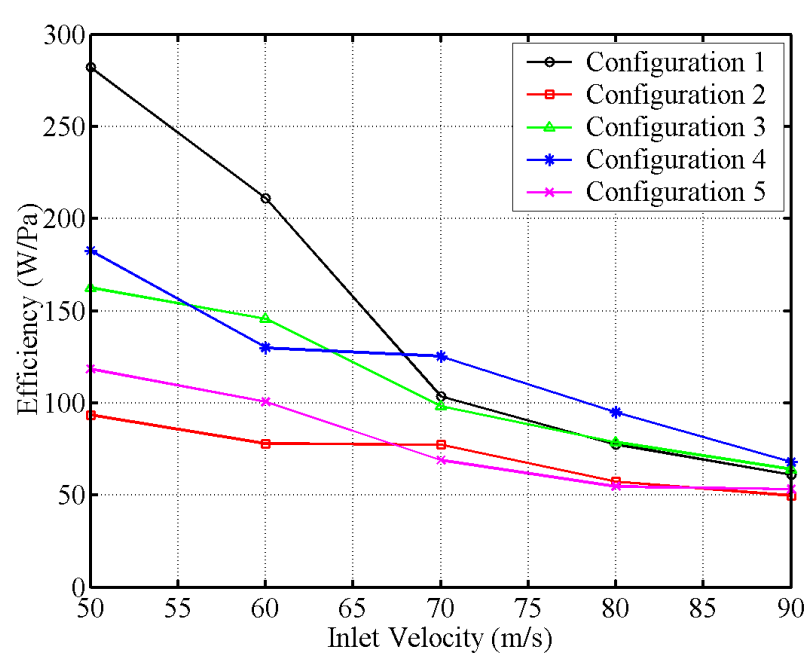

Figure 8: Efficiency of the investigated heat exchanger configurations.

uated as the efficiency of the heat exchanger configurations (see Figure 8). Configuration 1 and 4 yield the highest efficiency, because the charge air cooler is placed upstream of the radiators which allows for effective cooling of the radiators and the charge air cooler. Configurations 2 and 5 yield the lowest efficiency, because Configuration 2 has the lowest heat transfer rates and Configuration 5 has the highest pressure drop.

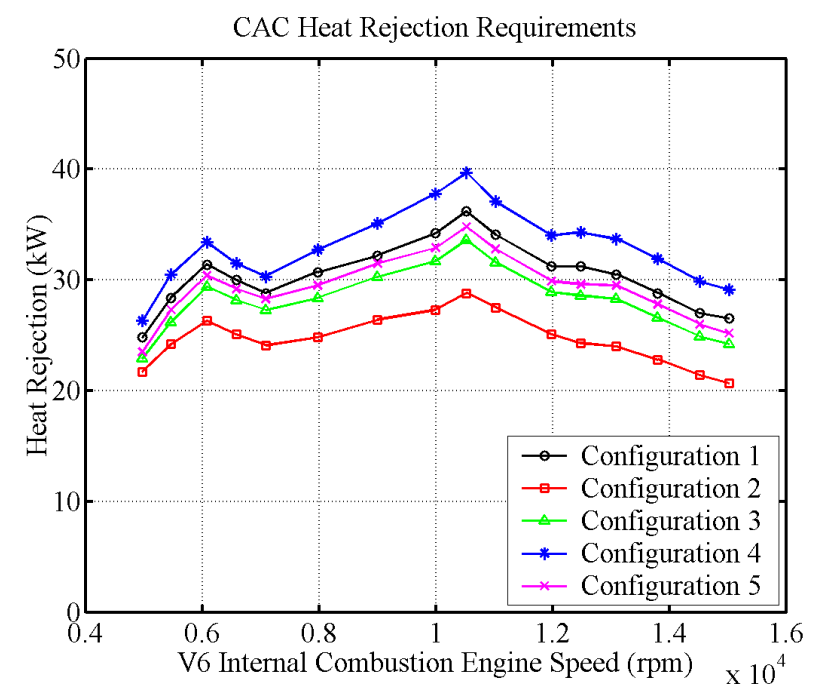

Figure 9: Charge air cooler (CAC) heat rejection $(k W)$ versus V6 internal combustion engine speed (rpm) correlation curves for each configuration.

The effect of various cooling configurations on the V6 internal combusion engine performance has been considered by using the AVL BOOST engine simulation software. The engine simulation has been performed based on the dimension and position of the charge air cooler by taking into account the efficiency of different cooling configurations. The charge air cooler heat rejection versus V6 internal combustion engine speed cor- 
relation curves for each configuration is shown in Figure 9, It can be seen again that Configuration 4 yields the highest heat rejection from the charge air cooler, because it has a larger inlet surface area with a minimum pressure drop and temperature increase of the cooling air before the radiators in the sidepod. To satisfy charge air cooler heat rejection requirements shown in Figure 9, the minimum air mass flow rate has to be considered as approximately $1.2 \mathrm{~kg} / \mathrm{s}$ for Configuration 4 (see Figure 6). To the heat rejection of $37.8 \mathrm{~kW}$, e.g. Configuration 4 required $0.3 \mathrm{~kg} / \mathrm{s}$ less air mass flow rate compared to Configuration 1 .

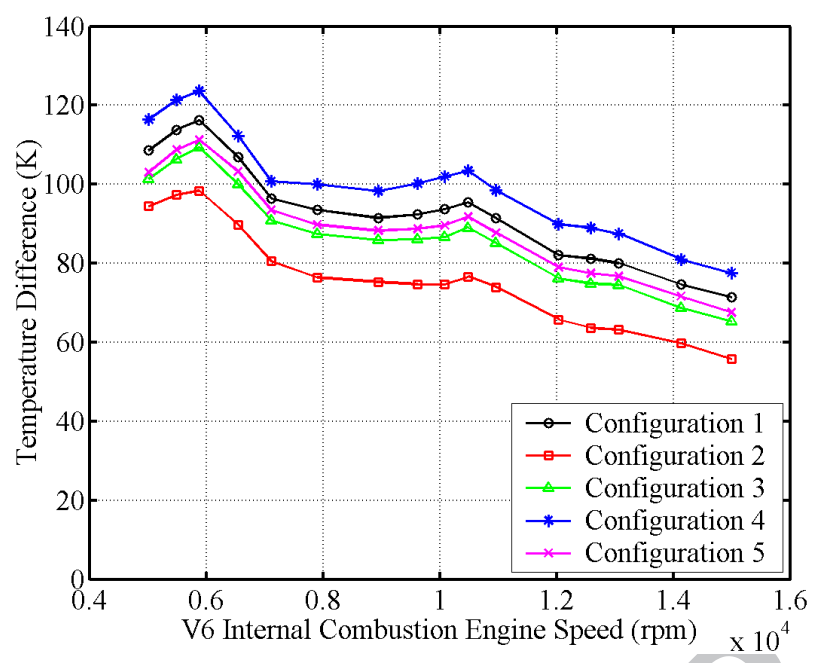

Figure 10: Temperature difference $(K)$ versus V6 internal combustion engine speed (rpm) correlation curves for each configuration.

To investigate the performance of various heat exchanger configurations through the simulation of the transitional air flow in the sidepod - which investigation is essentially related to sidepod design of the external cooling system of F1 engines wall temperature boundary conditions for the charge air cooler and the corresponding radiators have to be imposed. Temperature difference versus V6 internal combustion engine speed correlation curves for each configuration have been estimated by using the AVL BOOST software tool (see Figure 10. The importance of the investigation of the transitional air flow in an F1 car sidepod domain is that the effective cooling of the air will reduce the average running temperature of the engine, thus extending the life of the engine and its components. More details on the V6 engine design and simulation can be found in [35].

The variation in the numerical results between each configuration for the pressure drop is purely depending on the geometry and layout of each heat exchanger configuration. It can also be seen in Figure 11 that the iso-surfaces on the rearward facing section of the heat exchanger depict a snap shot of the sporadic separation. The minuscule buoyancy effects in the thermal boundary layer near the rear section of the tubes were observed to aid separation on the upward facing heated surfaces and suppress separation on the downward facing surfaces. In the cold air volumes between the cores, the Meredith effect [50] is present, because an air acceleration occurs as the air warms
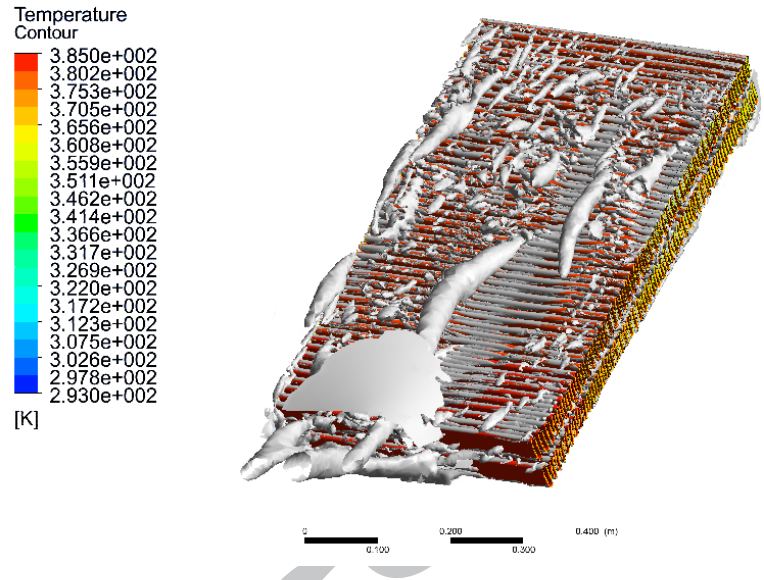

Figure 11: Vortical structures in the sidepod rear section with Q-criterion of 0.018 iso-surfaces.

up. This effect is caused by the decrease in air density which can be seen in the equations of the natural gas law and the conservation of momentum. The increase in the air temperature can be associated with a slight increase in air velocity which results in a different pressure drop behaviour compared to an adiabatic simulation, because the pressure drop is proportional to the velocity squared. The Meredith effect is also responsible for the variation in heat exchanger pressure drop which is observed between real and adiabatic tests in climatic chambers.

It is important to capture the physical effects of complex flow structures in a full car simulation, because the pressure gradients at the outlet duct of the sidepod influence the aerodynamic interaction between the sidepod flow, monkey seat, rear diffuser and rear wing. It has also an impact on the aerodynamic efficiency of the rear section and the resultant loading on the rear tyres depends on the vehicle speed and the yaw angle.

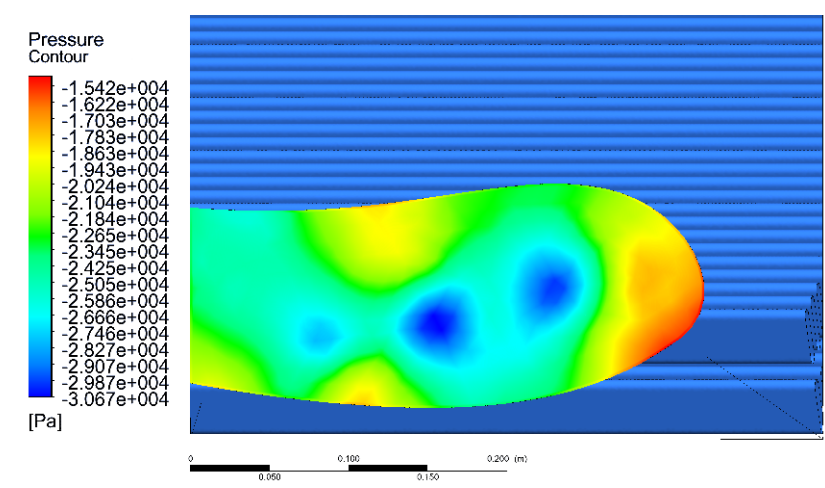

Figure 12: Outlet face pressure distribution relative to inlet face.

At the outlet duct of the generic sidepod shown in Figure 12 . the circular regions coloured blue are representative of lower pressure zones. These zones are associated with the centers of sustained vortices where the centrifugal forces of the rotating air reduce the pressure inside the vortex filament, which in turn induces centripetal acceleration of the fluid flow to sustain each vortex. These regions of lower pressure correspond to the core 
filaments and it can be confirmed by the iso-surface of the QCriterion vorticity 0.018 (see Figure 11). The central cores of these vortices usually have low turbulent intensity levels due to the viscous dissipation from the high relative velocities. The decrease in pressure is associated with a longitudinal acceleration of the flow along the length of the vortex filament.

The inlet and outlet shapes of the sidepods in formula one, and indeed most motorsport applications, generally do not take the form of the exhibited rectangular heat exchangers. In particular, the inlet duct shapes are optimised for external aerodynamic efficiency, which take into account the front tire wake, Y-250 vortices, barge boards, nose cone vortices, front suspension and other aerodynamics factors. Teams and suppliers collaborate to produce heat exchanger cores that conform to external aerodynamic requirements and internal packaging restrictions inherent in motorsport, however, there will inevitably be some non-uniformity of the flow velocity across the heat exchanger surfaces. In the present study, this problem has been depicted through the use of typical formula one shaped inlet and outlet ducts and a rectangular heat exchanger geometry. Due to the non-uniform volume expansion in the sidepod inlet domain, the resulting non-uniform pressure gradients induce lateral flow components in the front section of the sidepod, and turbulent vortices in the rear section of the side pod downstream of the cores. These flow characteristics are associated with aerodynamic inefficiencies and unwanted pressure drops across the sidepod ducts. The computational engineering approach presented in this paper enabled the simulation of the flow through the heat exchanger cores and the resulting sporadic separation events, vortices and outlet face pressure distributions. The high resolution of flow capture was made possible by the high density mesh and the Transitional SST turbulence model, whereas the use of the macroscopic heat exchanger model or porous media method might be too simplistic.

\section{Conclusions}

In this work, we presented a three-dimensional CFD approach which can be used in the holistic aerodynamics performance evaluation of various heat exchanger configurations. The aerodynamic effect on the rest of the vehicle can also be taken into account including the physically correct capturing of the Meredith effect and real non-adiabatic pressure drops across heat exchangers. This work provided numerical data to identify an optimum heat exchanger configuration for charge air coolers, which can be used in the design process of cooling systems. A detailed approach for numerically determining the pressure drop and heat transfer rate characteristics of a heat exchanger operating in transitional flow regime has also been provided. This was achieved by directly simulating the heat transfer from a core tube and theoretically incorporating the contribution of fins to heat transfer rates. As it is not possible to model thousands of fins in any CFD process and predict their effect on the heat exchanger performance, a theoretical approach was adopted to take into account the contribution of fins to the total heat rejection from each configuration, respectively.
This work includes a mesh refinement and a parametric study which led to the utilization of the Transitional SST engineering turbulence model and a very fine mesh with surface inflation to accurately capture boundary layer development and separation prediction in the transition flow regime. Note that six different computational meshes, instead of the usually employed three grid-levels, have been used to achieve grid independent numerical solutions and estimate the optimum number of cells for complex heat exchanger configurations. The flow fields can be accurately predicted by using a numerical solver to (1) resolve the boundary layer development in the transitional flow regime, (2) take into account the adverse pressure gradients on the rearward facing surfaces which induce separation, and (3) model correctly the convection and diffusion characteristics of the fluid flow. The evaluation of the effect of the heat exchanger positions relative to each other in the sidepod including investigations on the overall pressure drop and heat transfer performances have also been presented in this work. The full simulation of the sidepod domain and heat exchanger cores allows for valuable insight into the flow structures, which provide opportunities for further geometry optimization of the sidepod domain, heat exchanger configurations and the shape of core tubes, which could yield performance advantages worth a time benefit on-track. The insight provided by comprehensibly capturing the flow structures arising from the complex geometry allows improved physical representation of on-track flow behaviour in the CFD simulation environment. This aids physical data correlation as motorsport systems increase in complexity and numerical methods develop accordingly. This method of flow capturing can also be extended to the transient application of on-track behaviour of following another vehicle and real time effects of heat rejection, which governs the charged air inlet and power-unit coolant temperatures, respectively.

The highest performing configuration for multiple heat exchangers in a fixed sidepod domain has been determined as the Configuration 4. The placement of a thinner but wider charge air cooler upstream of the hotter fluid radiators results in an improved holistic surface to air temperature difference across the configuration, and a higher net heat transfer rate for an inlet mass flow rate range. A series stack arrangement with a temperature ascending hierarchy is determined to be optimal over a parallel stack arrangement for this application, the stated geometric constraints and boundary conditions. This solution could be used by motorsport teams in a bid to reduce the overall size of the heat exchanger cores to minimize the vehicle drag and maximize the power-unit waste heat rejection.

\section{Conflict of Interest}

None Declared.

\section{Acknowledgements}

The present research work was financially supported by the Centre for Advanced Vehicle Engineering and Centre for Computational Engineering Sciences at Cranfield University under 
project codes ETC1001E and EEB6001R, respectively. The authors would like to acknowledge the IT support for this research project by using High Performance Computing (HPC) facilities at Cranfield University, UK. The authors would also like to acknowledge the constructive comments of the reviewers of the Applied Thermal Engineering journal.

\section{References}

[1] J. Katz, Race car aerodynamics: Designing for speed, Bentley Publishers, USA, Cambridge (1995).

[2] R. Nimtan, A. K. Doost, N. Madani, Simulation of air flow under the hood of a passenger car using computational fluid dynamics, Research Journal of Applied Sciences, Engineering and Technology 6 (2013) 4583-4594.

[3] A. M. Hayes, J. A. Khan, A. H. Shaaban, I. G. Spearing, The thermal modeling of a matrix heat exchanger using a porous medium and the thermal non-equilibrium model, International Journal of Thermal Sciences 47 (2008) 1306-1315.

[4] M. M. A. Bhutta, N. Hayat, M. H. Bashir, A. R. Khan, K. N. Ahmad, S. Khan, CFD applications in various heat exchangers design: A review, Applied Thermal Engineering 32 (2012) 1-12.

[5] C.-C. Wang, R. L. Webb, K.-Y. Chi, Data reduction for air-side performance of fin-and-tube heat exchangers, Experimental Thermal and Fluid Science 21 (2000) 218-226.

[6] G. Xie, Q. Wang, B. Sunden, Parametric study and multiple correlations on air-side heat transfer and friction characteristics of fin-and-tube heat exchangers with large number of large-diameter tube rows, Applied Thermal Engineering 29 (2009) 1-16.

[7] R. Borrajo-Peláez, J.Ortega-Casanova, J. M. Cejudo-López, A threedimensional numerical study and comparison between the air side model and the air/water side model of a plain fin-and-tube heat exchanger, Applied Thermal Engineering 30 (2010) 1608-1615.

[8] J. P. Koplow, A fundamentally new approach to air-cooled heat exchangers, Sandia National Laboratories, Albuquerque, New Mexico 87185 and Livermore, California 94550, Sandia Report, SAND2009135192 (2009) 3-48.

[9] H. Fugmann, B. Nienborg, G. Trommler, A. Dalibard, L. Schnabel, Performance evaluation of air-based heat rejection systems, Energies 8 (2015) 714-741.

[10] A. Erek, B. Özerdem, L. Bilir, Z. İliken, Effect of geometrical parameters on heat transfer and pressure drop characteristics of plate fin and tube heat exchangers, Applied Thermal Engineering 25 (2005) 2421-2431.

[11] A. A. Bhuiyan, M. R. Amin, A. K. M. S. Islam, Three-dimensional performance analysis of plain fin tube heat exchangers in transitional regime, Applied Thermal Engineering 50 (2013) 445-454.

[12] A. A. Bhuiyan, M. R. Amin, R. Karim, A. S. Islam, Plate fin and tube heat exchanger modeling: Effects of performance parameters for turbulent flow regime, International Journal of Automotive and Mechanical Engineering 9 (2014) 1768-1781.

[13] Y.-J. Chang, C.-C. Wang, A generalized heat transfer correlation for louver fin geometry, International Journal of Heat and Mass Transfer 40 (1997) 533-544.

[14] J.-Y. Jang, L.-F. Hsu, J.-S. Leu, Optimization of the span angle and location of vortex generators in a plate-fin and tube heat exchanger, International Journal of Heat and Mass Transfer 67 (2013) 432-444.

[15] T. A. Cowell, M. R. Heikal, A. Achaichia, Flow and heat transfer in compact louvered fin surfaces, Experimental Thermal and Fluid Science 10 (1995) 192-199.

[16] C.-C. Wang, C.-J. Lee, C.-T. Chang, S.-P. Lin, Heat transfer and friction correlation for compact louvered fin-and-tube heat exchangers, International Journal of Heat and Mass Transfer 42 (1999) 1945-1956.

[17] M. E. Springer, K. A. Thole, Experimental design for flowfield studies of louvered fins, Experimental Thermal and Fluid Science 18 (1998) 258269.

[18] N. C. DeJong, A. M. Jacobi, Flow, heat transfer, and pressure drop in the near-wall region of louvered-fin arrays, Experimental Thermal and Fluid Science 27 (2003) 237-250.

[19] Z. gang Qi, J. ping Chen, Z. jiu Chen, Parametric study on the perform- ance of a heat exchanger with corrugated louvered fins, Applied Thermal Engineering 27 (2007) 539-544.

[20] C. T'Joen, H. Huisseune, H. Caniére, H. J. Steeman, A. Willockx, M. D. Paepe, Interaction between mean flow and thermo-hydraulic behaviour in inclined louvered fins, International Journal of Heat and Mass Transfer 54 (2011) 826-837.

[21] P. Gunnasegaran, N. H. Shuaib, M. F. A. Jalal, The effect of geometrical parameters on heat transfer characteristics of compact heat exchanger with louvered fins, ISRN Thermodynamics, doi:10.5402/2012/832708 2012 (2012) 1-10.

[22] G. C. Junjanna, N. Kulasekharan, H. R. Purushotham, Performance improvement of a louver-finned automobile radiator using conjugate thermal CFD analysis, International Journal of Engineering Research and Technology 1 (2012) 1-13.

[23] S. M. Salim, S. C. Cheah, Wall y+ strategy for dealing with wall-bounded turbulent flows, Proceedings of the International MultiConference of Engineers and Computer Scientists, Vol. II, IMECS 2009, March 18-20, Hong Kong, ISBN: 978-988-17012-7-5 (2009) 1-6.

[24] Y. Huang, R. Huang, X. Yu, F. LV, Simulation, experimentation, and collaborative analysis of adjacent heat exchange moduls in a vehicular cooling system, Journal of Zhejiang University - Science A (Applied Physics and Engineering), ISSN 1673-565X (Print); ISSN 1862-1775 (Online) 14 (2013) 417-426.

[25] C. T'Joen, A. Jacobi, M. D. Paepe, Flow visualisation in inclined louvered fins, Experimental Thermal and Fluid Science 33 (2009) 664-674.

[26] S. Grove, Engine cooling, Lecture Notes, Motorsport Structural Analysis Module, Cranfield University, UK (2014).

[27] J. P. Meyer, J. A. Olivier, Heat transfer and pressure drop characteristics of circular smooth tubes in the transitional flow regime, Proceedings of the 19th International Congress of Chemical and Process Engineering CHISA 2010 and the 7th European Congress of Chemical Engineering ECCE7, Prague, Paper: I6.1 (2010).

[28] J. P. Meyer, J. A. Olivier, Heat transfer in the transitional flow regime, Book Chapter 12 in Evaporation, Condensation and Heat Transfer, Edited by Amimul Ahsan, Published by InTech, Croatia (2011) 245-260.

[29] J. P. Meyer, J. A. Olivier, Transitional flow inside enhanced tubes for fully developed and developing flow with different types of inlet disturbances: Part i - adiabatic pressure drop, International Journal for Heat and Mass Transfer 54 (2011) 1587-1598.

[30] J. P. Meyer, J. A. Olivier, Transitional flow inside enhanced tubes for fully developed and developing flow with different types of inlet disturbances: Part ii - heat transfer, International Journal for Heat and Mass Transfer 54 (2011) 1598-1607.

[31] J. P. Meyer, J. Dirker, J. A. Olivier, D. V. Garach, The influence of different types of inlets on heat transfer of tubes in the transitional flow regime, Proceedings of International Conference on Advances in Mechanical Engineering, May 29-31, 2013, COEP, Pune, Maharashtra, India, Paper ICAME-2013/K3 (2013) 1-10.

[32] R. Sirwan, Y. Ali, L. C. Haw, S. Mat, A. Zaharim, K. Sopian, Modelling and optimization of heat transfer in smooth circular tube used in the shell and tube evaporator, Recent Advances in Fluid Mechanics, Heat and Mass Transfer and Biology, WSEAS, E-Library, ISBN: 978-1-61804065-7 (2012) 133-137.

[33] J. Hara, M. Iwasaki, I. Honda, Analysis of unsteady flow for vortex generator development for heat exchangers, Calsonic Kansei Technical Review 12 (2016) 89-105.

[34] Y. A. Cengel, A. J. Ghajar, Heat and mass transfer, fundamentals and applications, McGraw-Hill, Fifth Edition, New York (2011).

[35] T. Kempynck, The design and simulation of a F1 engine as defined by the 2014 regulations, Manuscript, Cranfield University, UK (2013).

[36] Y. A. Cengel, J. M. Cimbala, Fluid mechanics, fundamentals and applications, McGraw-Hill, New York (2006).

[37] G. Xie, B. Sunden, Q. Wang, L. Tang, Performance predictions of laminar and turbulent heat transfer and fluid flow of heat exchangers having large tube-diameter and large tube-row by artificial neural networks, International Journal of Heat and Mass Transfer 52 (2009) 2484-2497.

[38] C. Oliet, A. Oliva, J. Castro, C. D. Pérez-Segarra, Parametric studies on automotive radiators, Applied Thermal Engineering 27 (2007) 20332043.

[39] Z. Yang, J. Bozeman, F. Z. Shen, D. Turner, S. Vemuri, J. A. Acre, CFRM concept for vehicle thermal system, SAE Technical Paper Series (2002- 
01-1207), SAE 2002 World Congress, Detroit, Michigan, ISSN 01487191 (2002) 1-10.

[40] J. M. van Zyl, T. M. Harms, A. B. Taylor, Numerical investigation of the thermo-flow processes in an automobile underhood, Research and Development Journal of the South African Institution of Mechanical Engineering 25 (2009) 33-39.

[41] J.-Y. Jang, M.-C. Wu, Numerical and experimental studies of threedimensional plate-fin and tube heat exchangers, International Journal of Heat and Mass Transfer 39 (1996) 3057-3066.

[42] C.-C. Wang, K.-Y. Chi, Heat transfer and friction characteristics of plain fin-and-tube heat exchangers - part i: New experimental data, International Journal of Heat and Mass Transfer 43 (2000) 2681-2691.

[43] C.-C. Wang, K.-Y. Chi, C.-J. Chang, Heat transfer and friction characteristics of plain fin-and-tube heat exchangers - part ii: Correlation, International Journal of Heat and Mass Transfer 43 (2000) 2693-2700.

[44] J. Dong, J. Chen, Z. Chen, W. Zhang, Y. Zhou, Heat transfer and pressure drop correlations for the multi-louvered fin compact heat exchangers, Energy Conversion and Management 48 (2007) 1506-1515.

[45] R. Y. Miuraa, F. C. C. Galeazzo, C. C. Tadini, J. A. W. Gut, The effect of flow arrangement on the pressure drop of plate heat exchangers, Chemical Engineering Science 63 (2008) 5386-5393.

[46] J. Dong, J. Chen, W. Zhang, J. Hu, Experimental and numerical investigation of thermal-hydraulic performance in wavy fin-and-flat tube heat exchangers, Applied Thermal Engineering 30 (2010) 1377-1386.

[47] J. Dong, L. Su, Q. Chen, W. Xu, Experimental study on thermal-hydraulic performance of a wavy fin-and-flat tube aluminum heat exchanger, Applied Thermal Engineering 51 (2013) 32-39.

[48] Y.-L. He, W.-Q. Tao, Y.-W. Zhang, T. Xie, Analysis of heat transfer and pressure drop for fin-and-tube heat exchangers with rectangular winglettype vortex generators, Applied Thermal Engineering 61 (2013) 770-783.

[49] R. S. Doohan, P. K. Kush, G. Maheshwari, Exergy based optimization and experimental evaluation of plate fin heat exchanger, Applied Thermal Engineering 102 (2016) 80-90.

[50] L. Piancastelli, L. Frizziero, G. Donnici, The meredith ramjet: An efficient way to recover the heat wasted in piston engine cooling, ARPN Journal of Engineering and Applied Sciences 10 (2015) 5327-5333. 


\section{Highlights}

- Three-dimensional CFD transitional flow simulations in formula one car sidepods.

- Determination of an optimum heat exchanger configuration placed in the sidepods.

- Prediction of heat transfer and pressure drop to mass flow rate correlation curves.

- Performance of heat exchanger configurations for non-isothermal transitional flows.

- Overcome the difficulties of macroscopic heat exchanger and porous media methods. 


\section{Numerical investigation on various heat} exchanger performances to determine an optimum configuration for charge air cooler, oil and water radiators in $\mathrm{F} 1$ sidepods

Könözsy, László Z.

Elsevier

Pierre Salmon, László Könözsy, Clive Temple, Stuart Grove, Numerical investigation on various heat exchanger performances to determine an optimum configuration for charge air cooler, oil and water radiators in F1 sidepods, Applied Thermal Engineering, Volume 117, 5 May 2017, pp235-244, https://doi.org/10.1016/j.applthermaleng.2017.02.026 Downloaded from Cranfield Library Services E-Repository 